\title{
Inter-valley spiral order in the Mott insulating state of a heterostructure of trilayer graphene-boron nitride
}

\author{
Guo-Yi Zhu ${ }^{1}$, Tao Xiang ${ }^{2,3}$, and Guang-Ming Zhang ${ }^{1,3}$ \\ ${ }^{1}$ State Key Laboratory of Low-Dimensional Quantum Physics and \\ Department of Physics, Tsinghua University, Beijing 100084, China \\ ${ }^{2}$ Institute of Physics, Chinese Academy of Sciences, Beijing 100190, China \\ ${ }^{3}$ Collaborative Innovation Center of Quantum Matter, Beijing, 100084, China
}

(Dated: July 6, 2018)

\begin{abstract}
Recent experiment has shown that the ABC-stacked trilayer graphene-boron nitride Moire super-lattice at half-filling is a Mott insulator. Based on symmetry analysis and effective band structure calculation, we propose a valley-contrasting chiral tight-binding model with local Coulomb interaction to describe this Moire super-lattice system. By matching the positions of van Hove points in the low-energy effective bands, the valley-contrasting staggered flux per triangle is determined around $\pi / 2$. When the valence band is half-filled, the Fermi surfaces are found to be perfectly nested between the two valleys. Such an effect can induce an inter-valley spiral order with a gap in the charge excitations, indicating that the Mott insulating behavior observed in the trilayer graphene-boron nitride Moire super-lattice results predominantly from the inter-valley scattering.
\end{abstract}

Keywords: Moire superlattice, Mott phase, valley, Fermi surface nesting, multilayer graphene.

\section{INTRODUCTION}

The Moire super-lattice in the van der Waals heterostructure composed of multi-layer graphenes and hexagonal boron nitrides $(\mathrm{hBN})$ has recently attracted great interest ${ }^{116}$. Both graphenes and hBN have hexagonal lattice structures, but the original lattice periodicity is ruined due to the mismatch between their lattice constants. Nevertheless, the periodicity can be restored on a much larger length scale, i.e., the Moire wave length $\left(L_{\mathrm{M}} \simeq 15 \mathrm{~nm}\right)$, upon which a triangular Moire super-lattice emerges 24 . On the other hand, bilayer graphene with a small twisted angle can also form the Moire band structure ${ }^{7 / 10}$. In the magic-angle twisted bilayer graphene, the Moire bandwidth is reduced dramatically and the local Coulomb repulsion becomes relatively significant, leading to the observation of the Mott insulating state as well as the unconventional superconductivity around the half-filling ${ }^{11112}$. Meanwhile, it has been reported that a Mott insulating state also exists in the ABC-stacked trilayer graphene-hBN heterostructure ${ }^{13}$. In this experiment the low energy bandwidth is about $10 \mathrm{meV}$ while a Mott gap $\sim 2 \mathrm{meV}$ is observed at half filling. The comparable energy scale renders such a system in an intermediate coupling regime, therefore the role of band structure cannot be overemphasized.

In this paper, we will investigate the physical origin of the Mott insulating behavior observed in the trilayergraphene-hBN heterostructure. Based on the symmetry analysis and effective band structure calculation, we propose a minimal tight-binding model with local Coulomb interaction. This model defined on a triangular lattice characterizes an interacting electron system in a staggered fictitious magnetic field for each of the two degenerate valley degrees of freedom. By matching the van
Hove point positions of the effective low-energy bands, the staggered flux of each triangle is close to $\pi / 2$. At half-filling, the two valley Fermi surfaces are found to be perfectly nested. Such an effect leads to a novel correlated insulating state with an inter-valley spiral order and a charge excitation gap, giving a natural explanation to the experimental observation.

\section{MOIRE BAND STRUCTURE}

The ABC-stacked trilayer graphene (TLG) has the same Bravais lattice as in the monolayer graphene. But the electron and hole touching at zero energy support chiral quasiparticles with $3 \pi$ Berry phase, generalizing the low-energy band structure of the monolayer and bilayer graphene ${ }^{14}$. The $\mathrm{hBN}$ also forms a honeycomb lattice but has a lattice constant about $1.8 \%$ larger than that of the graphene. Thus the heterostructure of TLG and hBN can form a triangular Moire super-lattice shown in Fig. $1 \mathrm{a}$, which contains three interlaced regions. The region shaded by blue circles shows the maximal alignment between the TLG and hBN, denoted as the $\alpha$ zone; and the regions shaded by yellow or green triangles have a larger misalignment between the TLG and hBN, denoted as $\beta$ and $\beta^{\prime}$ zone, respectively. The $\beta$ zone differs from the $\beta^{\prime}$ zone by a sub-lattice exchange, defined by the $C_{6}$ rotation along the $z$-axis or the $M_{y}$ mirror reflection with respect to the $x-z$ plane. Each unit cell of the Moire super-lattice includes the $\alpha, \beta$ and $\beta^{\prime}$ zone. The TLG$\mathrm{hBN}$ heterostructure possesses the three-fold rotational symmetry along the $z$-axis $C_{3}$, the mirror reflection symmetry with respect to the $y$ - $z$ plane $M_{x}$, and the time reversal symmetry $\mathcal{T}$.

For both TLG and hBN, the honeycomb lattice can be 


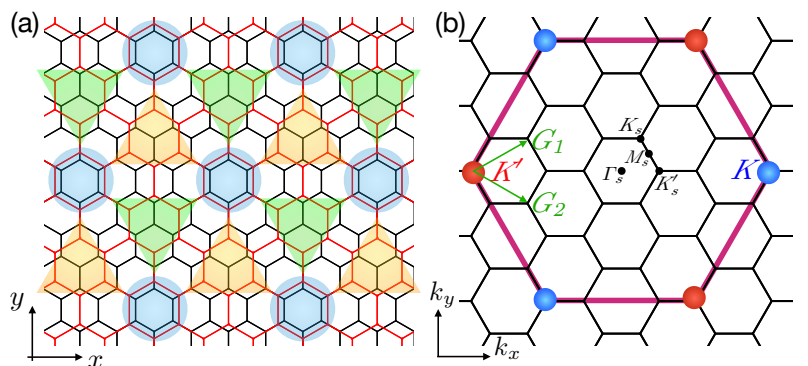

FIG. 1: Super-lattice structure and Brillouin Zone. (a) Superlattice formed by the TLG (black lines) and hBN (red lines). For the sake of clearness, we exaggerate the lattice constant mismatch to $33 \%$. The Moire pattern is composed of three interlaced regions shaded by blue, yellow, and green. (b) The Brillouin zone of the TLG on the original lattice (marked by the purple hexagon) is folded into many mini-Brillouin zone by the Moire periodic potential.

bipartitioned into two triangular sub-lattices. A Dirac cone is generated in the electronic structure at the charge neutral point (CNP). The Dirac fermions become massive when the sublattice symmetry $C_{2} \cdot \mathcal{T}$ which relates the two sub-lattices is broken $15 / 16$. In the hBN, boron and nitrogen atoms each form one of the sub-lattices, which breaks the symmetry between these two sub-lattices. This leads to a large energy gap (about $2.3 \mathrm{eV}$ ) in the low-lying excitations 15 . In contrast, the TLG itself is invariant under the sublattice symmetry, which protects the triple Dirac points near the Brillouin zone (BZ) corners. Thus the low-energy physics is dominated by the TLG, while $\mathrm{hBN}$ just contributes to a Moire scattering potential under second order perturbation. Such a Moire potential modulation folds the bands in the original BZ of the graphene layers into many mini-bands in the miniBrillouin zones (mBZ), as displayed in Fig. 1 b. As the $\mathrm{mBZ}$ is smaller by four order of magnitude than the original BZ, the bandwidth of the mini-bands is significantly suppressed. And the mini-bands near the charge neutral point (CNP) mainly originate from the low-energy valleys ( $K$ and $K^{\prime}$ shown in Fig. 1b) in the original TLG17.

There are two crucial points about the Moire modulation of the band structure. First, the two valleys originally connected within one band are now significantly separated into two degenerate bands, because the valley distance in the original BZ is significantly longer than the characteristic wave vector of the Moire potential. This is the reason why the valley degree of freedom enters into the superlattice as the internal degrees of freedom of electrons. Second, as the triple Dirac cones are in fact split by trigonal warping process in the TLG ${ }^{14}$, the splitting distance is relatively small in the original $\mathrm{BZ}$, but quite comparable to the scale of the folded mBZ (Fig. $2 \mathrm{a}$ and 2b). As a result, the flat dispersion between the Dirac cones dominate most area of the mBZ, which further suppresses the kinetic energy. Moreover, the Dirac point is gapped out by the interplay between the hBN and TLG,
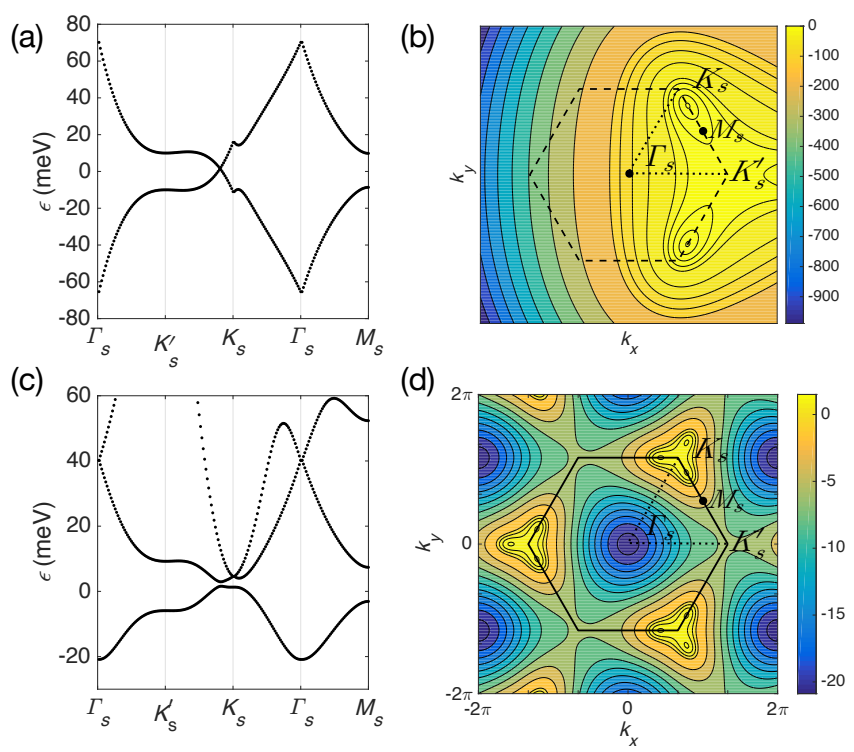

FIG. 2: Low-energy band structure and contour plot of the valence band. (a) Low-energy dispersion of the TLG without $\mathrm{hBN}$ is displayed in the proximity of the valley $K$ along the high symmetry lines of the mBZ. (b) Contour plot of the corresponding valence band near the CNP. The dashed black hexagon implies the $\mathrm{mBZ}$ once $\mathrm{hBN}$ is coupled to the TLG. The Dirac points are split away from $K_{s}^{\prime}$ and extend to almost the vicinity of $K_{s}$. Triple van Hove points gather near $K_{s}^{\prime}$ instead. (c) Low-energy Moire band structure for the valley $K$ whose Dirac points are close to $K_{s}$ in the mini-BZ. The Dirac points near $K_{s}$ are gapped out by the Moire potential. In obtaining this band structure, we have adopted the parameters used in Ref. ${ }^{14}$ and the Moire potential strength $80 \mathrm{meV}$ on the bottom layer of the TLG. (d) Contour plot of the corresponding valence band near the CNP in the $\mathrm{mBZ}$ (black hexagon). The vicinity of $K_{s}^{\prime}$ hosts three saddle points where the density of states diverges for this valley band. Color represents energy in unit of meV.

which breaks the sub-lattice symmetry. A valence band is thus separated from the other mini-bands by the Moire band gap (Fig. 22), which has four-fold degenerate associated with the spin and valley degrees of freedom. Electrons around the valleys $K$ and $K^{\prime}$ are related to each other by either one of the following transformations: the time-reversal symmetry $\mathcal{T}$, mirror reflection $M_{x}$, and $C_{6}$ rotation.

Using the effective two-component Hamiltonian for the $\mathrm{TLG}^{14}$, we have calculated the band structures with the Moire scattering potential $V_{\mathrm{M}}$ assumed to act only on the bottom graphene layer $\frac{13}{13}$. Since the two valley bands are connected through the mirror transformation $M_{x}$, we can just focus on the $K$ valley. In Fig. 2 2 , the electronic structure for the bands of valley $K$ is displayed. The contour plot of the corresponding valence band near the CNP is also shown in Fig. 2 $\mathrm{d}$. The triple Dirac points originally at $K_{s}^{\prime}$ are separated to locate along the boundary of the $\mathrm{mBZ}$ towards $K_{s}$, reducing the energy dispersion and inducing the triple van Hove singularity 
near $K_{s}^{\prime}$. When the Dirac points are further gapped out, the remaining triple van Hove points are the most remarkable fingerprint of the Moire band structure. More precisely, three van Hove points actually line along the mBZ boundary and center around the zone corner $K_{s}^{\prime}$. Increasing the value of $V_{M}$ pushes the three van Hove points towards $K_{s}^{\prime}$. Above all, due to the Moire scattering and the Dirac physics, the kinetic energy scale is quenched from 1 to $20 \mathrm{meV}$. Because the valence band is separated from the other bands, we are able to write a one-band minimal tight-binding model with valley and spin degeneracy.

Given the Moire mini-band structure, the minimal model should satisfy all the symmetries mentioned above, and reproduce the key feature of mini-valence-band: the triple van Hove points and ultra-flat dispersion. In the triangular Moire lattice sites labelled by $\alpha$ (Fig. 3a), one can effectively treat the two valleys as a pair of pseudospin denoted by the Pauli matrices $\tau_{a=x, y, z}$. Since the valley degrees are decoupled in the band folding, the nearest neighbor hopping should conserve the valley degrees of freedom. As the two valleys are related to each other by $M_{x}$ or $\mathcal{T}$, they are intrinsically born of chiral character. Indeed, the basic symmetries does not forbid the possibility of chiral flux. However, the time reversal symmetry $T$ requires the two valleys to have opposite flux phases, and the symmetry $M_{x}$ swaps both the hopping directions and valley degrees, which fixes the phases in the hopping integrals (Fig. 3a). Thus the minimal tight-binding model for the valence band of the TLG$\mathrm{hBN}$ heterostructure is given by the Hamiltonian

$$
H_{t}=-t \sum_{\mathbf{r}, \nu, \delta}\left(\mathrm{e}^{\mathrm{i} \nu \phi} c_{\mathbf{r}+\delta, \nu}^{\dagger} c_{\mathbf{r}, \nu}+\text { h.c. }\right)-\mu \sum_{\mathbf{r}, \nu} n_{\mathbf{r}, \nu},
$$

where $\delta=(1,0)$ and $(-1 / 2, \pm \sqrt{3} / 2)$ are the nearest neighboring vectors of the primitive unit cell, $\nu= \pm$ denote the valley indices, the fluxes alternate between the $\beta$ and $\beta^{\prime}$ triangles, and the hopping parameter $t$ measures effectively the valence bandwidth. For the simplicity, the spin degrees of freedom of electrons are frozen in our minimal tight-binding model. The flux penetrating each triangle is given by $\Phi=3 \nu \phi$, so this is a valleycontrasting chiral tight-binding model without breaking the time reversal symmetry.

In the momentum space, the band dispersion becomes

$$
\begin{aligned}
H_{t} & =\sum_{\mathbf{k}, \nu} c_{\mathbf{k}, \nu}^{\dagger} \epsilon_{\mathbf{k}, \nu} c_{\mathbf{k}, \nu}, \\
\epsilon_{\mathbf{k}, \nu} & =\varepsilon_{\mathbf{k}}^{e} \cos \phi-\nu \varepsilon_{\mathbf{k}}^{o} \sin \phi-\mu, \\
\varepsilon_{\mathbf{k}}^{e} & =-2 t\left(\cos k_{x}+2 \cos \frac{k_{x}}{2} \cos \frac{\sqrt{3} k_{y}}{2}\right), \\
\varepsilon_{\mathbf{k}}^{o} & =-2 t\left(\sin k_{x}-2 \sin \frac{k_{x}}{2} \cos \frac{\sqrt{3} k_{y}}{2}\right),
\end{aligned}
$$

where the electronic band dispersion $\epsilon_{k, v}$ generally varies
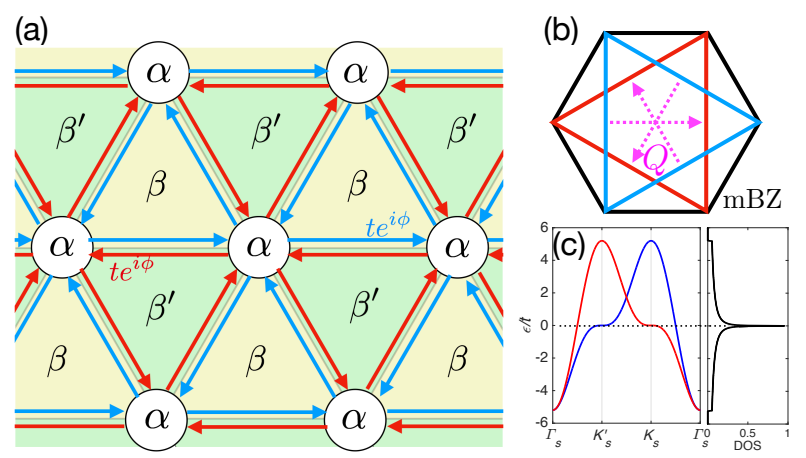

FIG. 3: Proposed minimal lattice model, Fermi surfaces and their band structure. (a) The TLG-hBN Moire superlattice composed of three different zones labelled by $\alpha, \beta$ and $\beta^{\prime}$, and the pattern of a valley contrasting staggered flux allowed by $C_{3}, M_{x}$, and $T$ symmetries. The $\alpha$ zones form the effective triangular lattice sites of the Moire superlattice. (b) The Fermi surfaces of two valleys (the red and blue triangles) at half-filling are nested by $\mathbf{Q}=(4 \pi / 3,0)$ and its equivalents connected by reciprocal unit vectors. (c) Band structures of the two valleys related by the $M_{x}$-symmetry and the local density of states.

with the flux phase $\phi$. By observing the band structure with varying $\Phi$, we noticed that the flux $\Phi$ essentially tunes those three $C_{3}$-related van Hove points. For the valley $K$, when $\Phi$ varies from 0 to $\pi / 2$, these van Hove points approach to $K_{s}^{\prime}$ along the mBZ boundary. Right at $\Phi=\pi / 2$, they merge into one triple van Hove point, as shown in the expansion around $K_{s}^{\prime}$

$$
\begin{aligned}
\epsilon_{\mathbf{k}+\mathbf{K}_{s}^{\prime},+}= & \left(-6+\frac{3}{2} k^{2}\right) \sin \left(\Phi-\frac{\pi}{2}\right) \\
& +\frac{1}{8}\left(k_{+}^{3}+k_{-}^{3}\right) \cos \left(\Phi-\frac{\pi}{2}\right)+O\left(k^{4}\right),
\end{aligned}
$$

where $k^{2} \equiv k_{x}^{2}+k_{y}^{2}$, and $k_{ \pm} \equiv k_{x} \pm \mathrm{i} k_{y}$. When $\Phi$ further increases, the triple van Hove point splits into three points along the line from $\Gamma_{s}$ to $K_{s}^{\prime}$ and its equivalents. So the minimal band structure calculated from the lowenergy effective band exhibits that three van Hove points are located right on the zone boundary in the vicinity of $K_{s}^{\prime}$, and hence the flux of the minimal model may be slightly smaller than $\pi / 2$. In principle, the exact value of $\Phi$ can be determined by matching the position of the van Hove points. In the following, we will focus on the ideal limit $\Phi=\pi / 2$, which reveals the essential correlated physics in the TLG-hBN heterostructure.

\section{INTER-VALLEY SPIRAL ORDER IN THE HALF-FILLED MOTT INSULATOR}

When $\Phi=\pi / 2$ at half-filling, the Fermi surface becomes a perfect triangle that touches the $\mathrm{mBZ}$ corners, as shown in Fig. 3p. In this case, the two Fermi sheets are perfectly nested and linked by three vectors 
$\mathbf{Q}=(4 \pi / 3,0)$ and $(-2 \pi / 3, \pm 2 \pi / \sqrt{3})$. However, these three nesting vectors are equivalent to each other, because they are simply related by the reciprocal vector of the mBZ. More explicitly, because of the particlehole symmetry $\epsilon_{\mathbf{k},+}=-\epsilon_{-\mathbf{k}-\mathbf{Q},+}$, we have the relation $\epsilon_{\mathbf{k},+}=-\epsilon_{\mathbf{k}+\mathbf{Q},-}$. In such a circumstance, the on-site Coulomb interactions become important. Taking into account the most relevant on-site Coulomb repulsion between valleys, we propose a valley version of the Hubbard model:

$$
H_{V}=V \sum_{\mathbf{r}} n_{\mathbf{r},+} n_{\mathbf{r},-},
$$

where $n_{\mathbf{r}, \nu}=\sum_{\sigma} c_{\mathbf{r}, \nu}^{\dagger} c_{\mathbf{r}, \nu}$ is the local electron density operator. The perfect Fermi surface nesting motivates us to introduce the following inter-valley order parameter

$$
\Delta_{\mathbf{Q}} \equiv V \sum_{\mathbf{k}}\left\langle c_{\mathbf{k}-\mathbf{Q},+}^{\dagger} c_{\mathbf{k},-}\right\rangle
$$

to decouple the Coulomb interaction $V$-term in Eq. (4) as

$$
H_{V} \simeq-\sum_{\mathbf{k}}\left(\Delta_{\mathbf{Q}} c_{\mathbf{k}+\mathbf{Q},-}^{\dagger} c_{\mathbf{k},+}+\text { h.c. }\right)+\frac{\Delta_{\mathbf{Q}}^{2}}{V}
$$

where $\Delta_{\mathbf{Q}}$ is a spatial uniform order parameter. Under the mean-field approximation, the above model Hamiltonian can be diagonalized, and the order parameter is determined by the self-consistent equation

$$
\int_{\mathrm{mBZ}} \frac{\sqrt{3} \mathrm{~d} k_{x} \mathrm{~d} k_{y}}{16 \pi^{2}} \frac{V}{\sqrt{\epsilon_{\mathbf{k},+}^{2}+\Delta_{\mathbf{Q}}^{2}}}=1,
$$

which is similar to the BCS gap equation. If we further assume that the overall $\mathrm{mBZ}$ contribution is dominated by a narrow shell of width $D$ around the Fermi energy, the solution to the above equation is then given

$$
\Delta_{\mathbf{Q}} \simeq D e^{-\frac{1}{V N(0)}},
$$

where $N(0)$ is the density of states at the Fermi level. At half-filling, $N(0)$ diverges, and an infinitesimal interaction $V$ can induce a finite inter-valley long-range order and gap out the Fermi surfaces completely. This has been confirmed by the numerical solution to the self-consistent equation, as shown in Fig. 4a. Actually this is a very peculiar insulating state induced by the inter-valley scattering $V$. In real space, $\Delta_{\mathbf{Q}}$ describes an inter-valley spiral long-range order of the valley pseudo-spin:

$$
\begin{aligned}
\left\langle\psi_{\mathbf{r}}^{\dagger} \tau_{x} \psi_{\mathbf{r}}\right\rangle & =\frac{2 \Delta_{\mathbf{Q}}}{V} \cos (\mathbf{Q} \cdot \mathbf{r}), \\
\left\langle\psi_{\mathbf{r}}^{\dagger} \tau_{y} \psi_{\mathbf{r}}\right\rangle & =-\frac{2 \Delta_{\mathbf{Q}}}{V} \sin (\mathbf{Q} \cdot \mathbf{r}), \\
\left\langle\psi_{\mathbf{r}}^{\dagger} \tau_{z} \psi_{\mathbf{r}}\right\rangle & =0
\end{aligned}
$$
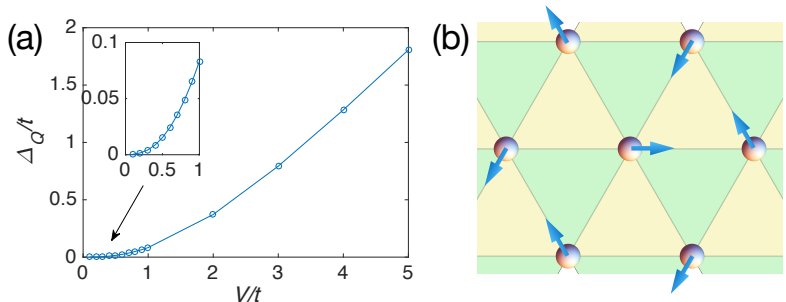

FIG. 4: The mean-field order parameter and its real space configuration. (a) Mean-field solution of the inter-valley spiral valley order parameter as a function of the on-site inter-valley interaction. (b) The real space configuration of the intervalley-spiral order.

with $\psi_{\mathbf{r}}^{\dagger}=\left(c_{\mathbf{r},+}^{\dagger}, c_{\mathbf{r},-}^{\dagger}\right)$, and the corresponding configuration is displayed in Fig. 4b. Therefore, it is this inter-valley spiral phase that describes the Mott insulating phase observed by the experiment in the TLG-hBN heterostructure ${ }^{13}$.

The nesting effect between two valley degrees of freedom successfully explains the Mott insulating phase at half-filling 13. Nevertheless, when the FS shows less prominent nesting, the weak coupling theory alone can hardly be justified. To probe the complete correlated physics, we will keep the band structure unchanged to explore the effective model Hamiltonian in the strong coupling limit.

\section{MOTT INSULATING PHASE IN THE STRONG COUPLING LIMIT}

Since the strong and weak coupling limits of the conventional one-band Hubbard model are adiabatically connected to each other, it can be expected that the correlated physics in the strong coupling regime is closely related with the weak coupling physics. By assuming $V \gg t$, we can treat the kinetic term as a perturbation. At zeroth order, the Hilbert space is separated into two Hubbard-like sub-bands by a charge Mott gap $\sim V$ at half filling. To pin down the many-body ground state, we need to resort to the second order perturbation. When the kinetic energy is regarded as a perturbation, the second order perturbation calculation leads to

$$
H_{J}=P_{1} H_{t} \frac{1-P}{-V} H_{t} P_{1},
$$

where $P_{1}$ restricts the local Hilbert space to that of one electron per lattice site $n=1$. The virtual hopping process between the nearest neighbor sites can induce an antiferro-valley exchange interaction:

$$
H_{J}=J \sum_{r, \delta} \mathbf{T}_{r} \cdot\left(\mathrm{e}^{\mathrm{i} 2 \phi T^{z}} \mathbf{T} \mathrm{e}^{-\mathrm{i} 2 \phi T^{z}}\right)_{r+\delta},
$$


where $J=4 t^{2} / V$ and the valley-pseudo-spin operators have been expressed by

$$
T_{r}^{a} \equiv \frac{1}{2} \sum_{\nu, \nu^{\prime}} c_{r, \nu}^{\dagger} \tau_{\nu, \nu^{\prime}}^{a} c_{r, \nu^{\prime}}, a=x, y, z
$$

Such an exotic valley-exchange interaction arises from the virtual hopping process between neighboring sites, which inherits the $\mathrm{SU}(2)_{v}$-breaking valley-contrasting flux.

It is known that, for the antiferromagnetic spin- $1 / 2$ Heisenberg exchange interaction on a triangular lattice, the Neel order along $S^{z}$ direction is frustrated by the lattice geometry and hence the spin moments are compromised to form a coplanar $120^{\circ}$ order. The antiferrovalley exchange interaction, however, carries a flux that can further lower the ground-state energy for the coplanar order. Apparently, as $\phi=\pi / 6$, each valley pseudospin in $H_{J}$ is rotated by $60^{\circ}$ before making the inner product with its neighbors, and hence every bond wins the most energy gain $-J / 4$ from the $120^{\circ}$ order. From another perspective, it is such a surprising coincidence that the flux $\phi=\pi / 6$ not only yields the qualitatively correct band structure to match the weak coupling theory, but also optimizes the ground state energy of the $120^{\circ}$ valley ordering Mott state from the strong coupling limit. It is remarkable to notice that this state is indeed adiabatically equivalent to the inter-valley spiral order at half-filling, when the charge degrees of freedom are frozen by the strong Coulomb repulsion. Both the weak and strong coupling theories thus point to the same Mott insulating state.

In the above analysis, we have neglected the spin degrees of freedom in our model formulation for the sake of clearness, because the singular Fermi surface structure strongly enhance the valley interaction only. When the spin degrees of freedom is retrieved, however, the corresponding insulating phase at quarter-filling is given by the spin-polarized antiferro-valley $120^{\circ}$ ordering state, while the half-filling insulating phase will be replaced by the spin-singlet antiferro-valley $120^{\circ}$ ordering state.

\section{DISCUSSION AND CONCLUSION}

Compared with the magic-angle twisted bilayer graphene ${ }^{11}$, the kinetic energy of both systems is suppressed by the band folding, resulting in a similar Moire super-lattice and the mBZ. The Dirac cones of the twisted bilayer graphene are separated and hybridized, yielding the van Hove singularity at $M_{s}$ point and flat dispersion in between. Similarly, in the TLG-hBN, the triple Dirac cones from the ABC stacked trilayer are separated by the trigonal warping with a strong hybridization, yielding the triple van Hove points and flat dispersion in between. The drastic distinction between these two systems is reflected in their symmetries and Fermi surface structures of the Moire bands. More precisely, with respect to the same Moire triangular super-lattice, the TLG-hBN is invariant under the symmetry $M_{x}$ while the twisted bilayer preserves the symmetry $M_{y} M_{z}$ instead. Consequently, the $C_{3}$-symmetric Fermi surfaces in the latter are distinct from that of the former by 30 degree rotation 10 . If the Fermi surfaces have a nesting effect, the three nesting vectors are no longer connected by the reciprocal lattice vector. Then the twisted bilayer graphene will be subjected to an inter-valley triple-Q nesting, and the inter-valley Coulomb repulsion induces a drastically distinct Mott insulating phase.

What experimental signature could be observed for this order? In the large length scale of Moire super-lattice characteristic of $\lambda_{M}=15 \mathrm{~nm}$, where the valley degrees of freedom are treated as the internal degrees of freedom inside each Moire supercell, the order with long-range wave vector $\mathbf{Q}$ exhibits an in-plane spiral feature of the valley pseudo-spins, but does not involve a density spatial modulation running over the Moire superlattice. Nevertheless, when zooming into the small length-scale of the original graphene lattice characteristic of $a=0.246 \mathrm{~nm}$, the valley degrees of freedom retain their orbital character. The nesting between the two valleys in the original $\mathrm{BZ}$ gives rise to a short range nesting wave vector, corresponding to a $\sqrt{3} a \times \sqrt{3} a$ charge modulation pattern in the microscopic lattice.

Moreover, in the magic-angle twisted bilayer graphene, unconventional superconductivity was also observed slightly away from the half-filling ${ }^{12}$. Naturally, one would ask whether this TLG-hBN heterostructure could also become a superconductor by doping away from the halffilling. If yes, what is the most probable pairing symmetry. From our above analysis, the inter-valley scattering should still be the most important channel of pairing interactions, because the intra-valley pairing is not energetically favored due to the peculiar Fermi surface structures. If only the inter-valley Coulomb repulsion is considered, there is no privilege between spin singlet and spin triplet pairing. However, the inter-valley Hund's rule coupling favors a spin-triplet pairing state. Therefore, the superconducting state in the TLG-hBN is expected to be in the inter-valley spin-triplet pairing channel. A detailed discussion on this will be given in a separate paper.

In conclusion, we have proposed a minimal tightbinding model to describe the low-energy states of the TLG-hBN super-lattice. Compared to the low-energy effective bands, the valley-contrasting staggered flux of each Moire triangle acquires the value of $\pi / 2$. At halffilling, the Fermi surfaces are perfectly nested between the two valleys. This leads to a strong inter-valley scattering and the system becomes unstable against an intervalley spiral order. We believe that this inter-valley spiral ordered phase is just the Mott insulating phase observed in the experiments ${ }^{13}$.

Note added: while in the preparation of this work, we noticed that two preprint\$19/20 on the model for magicangle twisted bilayer graphene appear. One of them 19 proposed a similar tight-binding model for the TLG-hBN 
heterostructure.

Conflict of interest The authors declare that they have no conflict of interest.

Acknowledgments: This work was supported by the National Key Research and Development Program of MOST of China (2017YFA0302900) and the National Natural Science Foundation of China (11474331).

\section{APPENDIX}

The low-energy band structure of the TLG is composed of A-sublattice on the bottom layer $\left(A_{1}\right)$ and B-sublattice on the top layer $\left(B_{3}\right)$, while the other sublattices are bonded by the on-site interlayer coupling $\gamma_{1}=0.39 \mathrm{eV}$ and belong to the high-energy sector $\frac{1418}{18}$. Therefore, a low-energy effective Hamiltonian on the two-layer triangular lattice that accounts for the TLG:

$$
\hat{H}_{\mathrm{ABC}}^{\mathrm{eff}}=\int d^{2} k \psi_{\mathbf{k}}^{\dagger} H_{\mathrm{TD}}(\mathbf{k}) \psi_{\mathbf{k}}
$$

where $\psi_{\mathbf{k}} \equiv\left(A_{1, \mathbf{k}}, B_{3, \mathbf{k}}\right)^{T}$ is a two-component spinor. The warped triple Dirac Hamiltonian matrix is given by

$$
\begin{aligned}
H_{\mathrm{TD}}(\mathbf{k})= & \frac{v_{0}^{3}}{\gamma_{1}^{2}}\left(\begin{array}{cc}
0 & \left(\pi^{\dagger}\right)^{3} \\
\pi^{3} & 0
\end{array}\right)+\frac{2 v_{0} v_{4} k^{2}}{\gamma_{1}}\left(\begin{array}{ll}
1 & 0 \\
0 & 1
\end{array}\right) \\
& -\left(\frac{2 v_{0} v_{3} k^{2}}{\gamma_{1}}-\frac{\gamma_{2}}{2}\right)\left(\begin{array}{ll}
0 & 1 \\
1 & 0
\end{array}\right),
\end{aligned}
$$

where $\pi=k_{x}+i k_{y}$ and $v_{i}=\sqrt{3} \gamma_{i} a / 2(i=0,1,2,3,4)$. The triple Dirac dispersion is inherited from the three layers of graphene. On the other hand, the $\mathrm{hBN}$ has a large atomic energy $0.8 \pm 2.5 \mathrm{eV}$, and can therefore be integrated out, leaving a Moire potential contribution to the TLG ${ }^{15}$ :

$$
\hat{H}_{\text {Moire }}=V_{M} \sum_{r, G} e^{i G \cdot r} B_{3, r}^{\dagger} B_{3, r}=V_{M} \sum_{\mathbf{k}, G} B_{3, \mathbf{k}-G}^{\dagger} B_{3, \mathbf{k}},
$$

where $G=\frac{4 \pi}{\sqrt{3}}\left(\sin \frac{\pi j}{3}, \cos \frac{\pi j}{3}\right)$ with $j=1,2, \ldots 6$. In the momentum space, $\hat{H}_{\text {Moire }}$ essentially scatters the Dirac cones by the vector $G$, iteration of which gives rise to the Moire reciprocal lattice. Therefore, the Bloch Hamiltonian in the $\mathrm{mBZ}$ can be obtained as

$$
\begin{aligned}
\hat{H}_{e f f}(\mathbf{k})= & \sum_{\mathbf{q}} \psi_{\mathbf{k}-\mathbf{q}}^{\dagger} H_{\mathrm{TD}}(\mathbf{k}-\mathbf{q}) \psi_{\mathbf{k}-\mathbf{q}} \\
& +\sum_{\mathbf{q}, G} \psi_{\mathbf{k}-\mathbf{q}-\mathbf{G}}^{\dagger}\left(\begin{array}{cc}
0 & 0 \\
0 & V_{M}
\end{array}\right) \psi_{\mathbf{k}-\mathbf{q}}
\end{aligned}
$$

where $\mathbf{q}=m G_{1}+n G_{2}$ denotes the reciprocal lattice site. By exact diagonalizing $\hat{H}_{e f f}(\mathbf{k})$, we obtain the lowenergy mini-band structure of the TLG-hBN heterostructure. The calculation result in this paper is performed by truncating $-5 \leq m \leq 5$ and $-5 \leq n \leq 5$. Besides, we perform the Fourier transform to change the Bloch wave function to the real space for each momentum $\mathbf{k}$ :

$$
\Psi_{\mathbf{k}}^{\dagger}=\sum_{\mathbf{q}}\left(u_{\mathbf{q}}^{\mathbf{k}} A_{1, \mathbf{k}-\mathbf{q}}^{\dagger}+v_{\mathbf{q}}^{\mathbf{k}} B_{3, \mathbf{k}-\mathbf{q}}^{\dagger}\right)
$$

Then from the probability distribution $\left|\sum_{\mathbf{q}} u_{\mathbf{q}}^{\mathbf{k}} e^{-i \mathbf{q} \cdot r}\right|^{2}+$ $\left|\sum_{\mathbf{q}} v_{\mathbf{q}}^{\mathbf{k}} e^{-i \mathbf{q} \cdot r}\right|^{2}$, the summation over the valence band gives rise to the local density of states (LDOS):

$$
\rho(x)=\int_{\mathrm{mBZ}} \frac{\sqrt{3} d^{2} k}{8 \pi^{2}}\left|\sum_{\mathbf{q}} u_{\mathbf{q}}^{\mathbf{k}} e^{-i \mathbf{q} \cdot r}\right|^{2}+\left|\sum_{\mathbf{q}} v_{\mathbf{q}}^{\mathbf{k}} e^{-i \mathbf{q} \cdot r}\right|^{2} .
$$

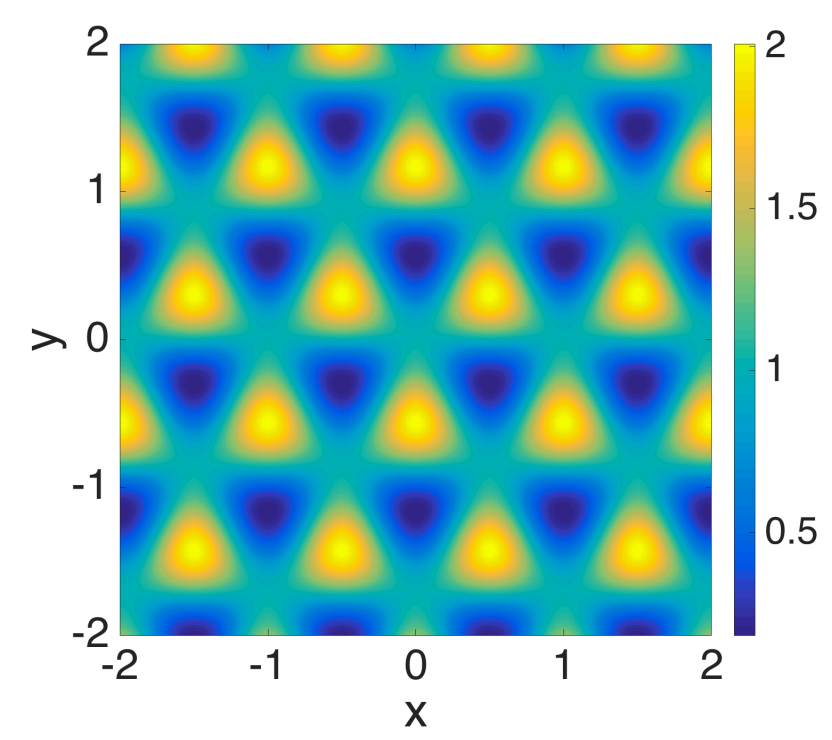

FIG. 5: The normalized local density of states corresponding to the valence band. $\mathrm{x}$ and $\mathrm{y}$ axes are in the unit of Moire wavelength, and the Moire scattering potential is assumed $V_{M}=80 \mathrm{meV}$.

Since we are mainly concerned with the valence band, which is well separated from the conductance band, we do not distinguish the two layer degrees of freedom. Otherwise there would be a two band model instead. As the two valley degrees of freedom are related via the mirror symmetry $M_{x}$, they have the same LDOS distribution, which matches a triangular lattice. Although the maximum of the LDOS is shifted to $\beta$ zones, the LDOS is not at all depleted in $\alpha$ zones. The Wannier function is therefore located on either $\alpha$ or $\beta$ zone. The tight-binding model of the low-energy valence band should therefore be defined on a triangular lattice with two valleys on each site. Without the sublattice degrees of freedom, it leads to an equivalent minimal model whether the Wannier center is located on the $\alpha$ or $\beta$ zone, as both of them share the same symmetries $C_{3}$ and $M_{x}$. For the symmetric reason, we have assumed that the Wannier center is located on the $\alpha$ zones. 
1 M. Yankowitz, J. Xue, D. Cormode, et al. Emergence of super-lattice Dirac points in graphene on hexagonal boron nitride, Nat Phys, 8:382-386 (2012).

2 C. R. Dean, L. Wang, P. Maher, et al. Hofstadter's butterfly and the fractal quantum Hall effect in moire superlattices, Nature, 497:598-602 (2013).

3 B. Hunt, J. D. Sanchez-Yamagishi, A. F. Young, et al. Massive Dirac fermions and Hofstadter butterfly in a van der Waals heterostructure, Science, 340: 1427-1430,(2013).

${ }^{4}$ L. A. Ponomarenko, R. V. Gorbachev, G. L. Yu, et al. Cloning of Dirac fermions in graphene superlattices, Nature, 497: 594-597 (2013).

5 W. Yang, G. Chen, Z. Shi, et al. Epitaxial growth of singledomain graphene on hexagonal boron nitride, Nat Mater, 12: $792-797$ (2013).

6 Z. Shi, C. Jin, W. Yang, et al. Gate-dependent pseudospin mixing in graphene/boron nitride moire superlattices, Nat Phys 10: 743-747 (2014).

7 J. M. B. L. D. Santos, N. M. R. Peres, A. H. C. Neto, Graphene bilayer with a twist: electronic structure, Phys Rev Lett 99:256802 (2007).

8 R. Bistritzer, A. H. MacDonald, Moire bands in twisted double-layer graphene, Proc Natl Acad Sci USA, 108: 1223-12237 (2011).

9 G. Trambly de Laissardiere, D. Mayou, L. Magaud, Numerical studies of confined states in rotated bilayers of graphene, Phys Rev B 86: 125413 (2012).

10 Y. Cao, J. Y. Luo, V. Fatemi, et al. Superlattice-induced insulating states and valley-protected orbits in twisted bilayer graphene, Phys Rev Lett 117: 116804 (2016).

11 Y. Cao, V. Fatemi, A. Demir, et al. Correlated insulator behaviour at half-filling in magic-angle graphene superlattices, Nature 556: 80-84 (2018).

12 Y. Cao, V. Fatemi, S. Fang, et al. Magic-angle graphene superlattices: a new platform for unconventional superconductivity, Nature 556: 43-50 (2018).

13 G. Chen, L. Jiang, S. Wu, et al. Gate-tunable Mott insulator in trilayer graphene-boron nitride Moire superlattice, arXiv:1803.01985

14 M. Koshino, E. McCann, Trigonal warping and Berry's phase $N \pi$ in ABC-stacked multilayer graphene, Phys Rev B 80: 165409 (2009).

15 M. Kindermann, B. Uchoa, D. L. Miller, Zero-energy modes and gate-tunable gap in graphene on hexagonal boron nitride, Zero-energy modes and gate-tunable gap in graphene on hexagonal boron nitride, Phys Rev B, 86: 115415 (2012).

16 Y. Kim, B. J. Wieder, C. L. Kane, et al. Dirac line nodes in inversion-symmetric crystals, Phys Rev Lett 115: 036806 (2015)

17 J. R. Wallbank, A. A. Patel, M. Mucha-Kruczynski, et al. Generic miniband structure of graphene on a hexagonal substrate, Phys Rev B, 87: 245408 (2013).

18 F. Zhang, B. Sahu, H. K. Min, et al. Band structure of ABC-stacked graphene trilayers, Phys Rev B, 82: 035409 (2010).

19 H. C. Po, L. Zou, A. Vishwanath, et al. Origin of Mott insulating behavior and superconductivity in twisted bilayer graphene, arXiv:1803.09742.

20 N. F. Q. Yuan, Liang Fu, A model for metalinsulator transition in graphene superlattices and beyond, arXiv:1803.09699 